\title{
Beitrag zum anatomischen Verhalten der Schleim- haut der Cervix und des Uterus während der Schwangerschaft und im Frïhwochenbett.
}

\author{
Von \\ Dr. Kroenig, Privatdocent in Leipzig.
}

(Mit 7 Abbildnngen auf Tafel I u. II.)

Ueber das anatomische Verhalten der Schleimhaut des Uterus während der Schwangerschaft und im Frühwochenbett liegen die vorzüglichen Arbeiten von Leopold, Langhans, Marchand, PelsLeusden, Aschoff, Hofmeier, Franqué, Gebhard, Klein, Ries, Strahl und vielen Anderen vor. Ich ${ }^{1}$ ) habe ebenfalls über verschiedene anatomische Einzelheiten berichtet in meiner Monographie: „Bakteriologie des weiblichen Genitalcanales." Der Zweck der nachfolgenden Arbeit ist vor Allem der, einige Abbildungen von mikroskopischen Präparaten der Cervix und der Uterusschleimhaut während der Schwangerschaft und im Wochenbett zu bringen. In der grundlegenden Arbeit von Leopold2) in diesem Archiv, 1877, sind schon verschiedene Abbildungen von der Uterusschleimhaut im Wochenbett gegeben, doch geben die dort reproducirten Bilder nur eine allgemeine schematische Uebersicht, während sie über die Einzelheiten des Verhaltens der Schleimhaut nur wenig Aufklärung geben. Leopold hat seinen Bildern eine vorzügliche Beschreibung der gewonnenen Befunde beigefügt, welche ich auf Grund meiner Untersuchungen in der früher erwähnten Arbeit im Allgemeinen nur bestätigen konnte.

1) Bakteriologie des weiblichen Genitalcanals von Menge und Kroenig. Theil II: Kroenig, Bakteriologie des Genitalcanals d. schwangeren, kreissenden und puerperalen Frau. S. $187 \mathrm{ff}$.

2) Leopold, Studien über die Uterusschleimhaut während d. Menstruation, Schwangerschaft und Wochenbett. Dieses Archiv. Bd. XII. S. 170. 
Seit dieser Arbeit von Leopold finden wir später nirgends genauere Abbildungen der Schleimhaut der Cervix und des puerperalen Uterus, so dass in vielen Lehrbüchern der Geburtshülfe meistens diese Uebersichtsbilder von Leopold übernommen sind, die nach meiner Meinung, wenn die präcise Darstellung von Leopold fehlt, falsche Anschauungen erwecken könnien. Da die hier vorliegenden Abbildungen gewissermassen eine Ergänzung der Leopold'schen Wiedergaben darstellen, so hat sich die Verlagsbuchhandlung bereit erklärt, an dieser Stelle einige Reproductionen meiner Präparate zu bringen; für dieses Entgegenkommen sage ich hiermit meinen Dank.

Ich habe mich bei der Beschreibung der Präparate kurz gefasst, weil ich zum Theil dieselben schon in der oben eitirten Monographie ausführlicher wiedergegeben habe. Ich möchte an dieser Stelle nur noch einmal darauf hinweisen, wie wichtig es ist, für derartige Präparate nur frisch aus der Leiche entnommenes und gut conservirtes Material zu verwerthen. Ich war durch die Freundlichkeit meines früheren Chefs, Herrn Geh.-Raths $\mathrm{Z}$ weifel, in der Lage, die Präparate gewöhnlich unmittelbar post mortem der Leiche entnehmen zu können, um sie dann direct in der Härteflüssigkeit zu fixiren. In fast allen Fällen wählte ich die von Zenker empfohlene Sublimat-Chromsäuremischung; welche sich nach unserer Erfahrung zur. Festhaltung des Epithelbelags der Drüsen besonders gut eignet. Es ist dies für die hier in Frage kommenden Präparate deswegen von Bedeutung, weil gerade am Ende der Scbwangerschaft die Drüsenräume der Decidua von einem plattgedrückten kubischen Epithel bedeckt sind, welches nur dann noch im mikroskopischen Bilde sichtbar zu machen ist, wenn das Präparat sehr bald post mortem in sorgfältigster Weise gehärtet ist. Angaben von vollständig fehlendem Epithelbelag auch in den tieferen Theilen der Uterusschleimhaut sind zum Theil unseres Erachtens auf nicht frisch gewonnenes Material oder auf schlechte Conservirung zurückzuführen.

Nach diesen kurzen Vorbemerkungen gehe ich direct zur Beschreibung der wiedergegebenen Abbildungen über.

Verhalten der Cervix uteri am Ende der Schwangerschaft (Abbildung 1).

Das Präparat stammt von der erstgeschwängerten H., 19 Jahre alt. Die Schwangere war wegen häufiger Anfälle von Herz- 
schwäche schon in der Mitte des 9. Schwangerschaftsmonats in die Anstalt eingetreten. Einige Tage nach der Aufnahme trat plötzlich bei ihr eine acute Herzschwäche ein, welche auch durch Kampherinjectionen nicht behoben werden konnte. Da das Kind lebensfähig war, wird in der Agone der Kaiserschnitt ausgeführt und ein frischtodtes Kind extrahirt. Der Tod der Mutter erfolgt bei der Operation, und es wird, während die Placenta noch theilweise an der Wand anhaftet, gleich die Exstirpation des Uterus angeschlossen.

Die Section ergiebt eine ulceröse Endocarditis, Lungenödem und im rechten unteren Lungenlappen einen käsigen, zum Theil verkalkten Herd, welcher in schwieliges Gewebe eingebettet ist.

Wehen waren bei der Schwangeren noch nicht eingetreten.

Das Präparat wird in der Weise behandelt, dass durch einen circulären Schnitt die ganze Cervix und der untere Theil des Uterus mit anhaftenden Eihäuten von dem Corpus uteri abgetrennt und für sich allein in Zenker'scher Flüssigkeit gehärtet wird. Ein Frontalschnitt durch das gehärtete Präparat zeigt, dass der Cervikalcanal noch als cylindrischer Canal in einer Länge von $1,8 \mathrm{~cm}$ erhalten ist. Im Cervikalcanal sitzt ein zäher Schleimpropf. Die Wand des Cervikalcanals besteht, wie in dem Uebersichtsbild $I_{3}$ dargestellt ist, aus stark gebuchteten Plicae palmatae. Bei a ist der Cervikalcanal flach trichterförmig erweitert und in die Fruchthöhle eingezogen. Vom Müller'schen Ring (a) bis zur Eihaftstelle (b) beträgt die Entfernung im gehärteten Präparat 1,1 cm. Diese letztere Stelle habe ich, da sie infolge der frühen Gewinnung des Präparates sehr schön erhaltenes Gewebe zeigt, in der Abbildung $\mathrm{I}_{1}$ wiedergegeben in Vergrösserung Zeiss' Objectiv $a_{2}$, Ocular 2.

In dem Präparat ist bei a etwas weiter links und im Bilde nicht mehr sichtbar der Müller'sche Ring, bei b die Eihautansatzstelle an der Vera; d ist die über dem Cervikalcanal ausgespannte Eihaut mit dem wohlerhaltenen, stark ausgeprägten Amnionepithel. Es entspricht dieses Präparat im Wesentlichen einer Beschreibung, welche Marchand ${ }^{1}$ ) und Küstner ${ }^{2}$ ) in diesem Archiv geben.

1) Marchand, Noch einmal das Verhalten der Cervix uteri in der Schwangersohaft. Dieses Archiv. S. $169 \mathrm{ff}$.

2) Küstner, Beitrag zur Anatomie der Cervix uteri während der Schwangerschaft und des Wochenbetts. Dieses Archiv. Bd. XII. S. $383 \mathrm{ff}$. 
Ich möchte aber auf folgende Einzelheiten besonders noch aufmerksam machen. Die Strecke von a bis b ist, wie eine bei stärkerer Vergrösserung (Ocular 2, Objectiv E) wiedergegebene Stelle bei $\mathrm{x}$ in der beigegebenen Skizze $\mathrm{I}_{2}$ zeigt, überall mit hohem Cylinderepithel und basal gestellten Kernen bekleidet. Bei b sehen wir das Epithel sich auf die freie Fläche der über den Cervikalcanal gespannten Eihaut fortsetzen, sich dabei sehr stark abflachend, doch ist es in der Gegend von $\mathrm{x}$ immer noch als ein deutlicher Epithelbelag mit niedrigen, kubischen Epithelzellen sichtbar (vergl. $I_{2}$ ). Ob dieses Epithel als ein fortgesetztes Cervixepithel oder Uterusepithel zu betrachten ist, lasse ich dahingestellt. Von $f$ aus hat sich auf die freien Eihäute zum Theil Decidua reflexa fortgesetzt, welches besonders deutlich bei $\mathrm{c}$ in der $\mathrm{Ab}$ bildung sichtbar. ist. Sehr schön ist aus der Abbildung ausserdem die breite Lage von Chorionepithel (e) und das durch die Entleerung der Eihöhle stark gefaltete Amnion (d) zu erkennen.

Während sich mit Epithel ausgekleidete Drüsenräume auf dem Bilde $I_{1}$ zum Theil noch unterhalb der Eihautabgangsstelle nach dem Cervikalcanal hin nachweisen lassen, setzt. sich die Decidua vera, welche bei $f$ als eine aus dichtgedrängten. Deciduazellen zusammengesetzte, deutlich markirte Schicht zu sehen ist, nur wenig auf den nach dem Cervikalcanal hin gelegenen Theil fort, sondern hier haben wir die theils runden, theils spindelförmigen Zellen des Cervixgewebes, durchbrochen von den zahlreichen, im Bilde sichtbaren Blutcapillaren der Cervix. Mit Berücksichtigung der orientirenden Skizze $I_{3}$ zeigt daher dies Präparat, wie bei Erstgeschwängerten während der Gravidität unter dem Einfluss von Schwangerschaftswehen eine Entfaltung der oberen Cervixabschnitte stattfindet. Wenn auch das Präparat nicht für die Frage nach dem Contractionsringe direct verwerthbar ist, so scheint mir doch das scharfe Absetzen der Decidua vera an der Stelle, wo die Eihäute von der Uteruswand sich ablösen und über dem Cervikalcanal sich ausspannen, ein gewisser Wahrscheinlichkeitsbeweis mit dafür zu sein, dass eine Umwandlung der Cervicalschleimhaut in Decidua vera während der Schwangerschaft nicht stattfindet und $n$ dass die entfalteten Theile während der Schwangerschaft ihre Cervixschleimhaut bewahren und Alles, was mit Decidua bekleidet ist, anatomisch zum Corpus uteri gehört"i).

1) Gebhard, Pathologische Anatomie der weiblichen Sexualorgane. Leipzig. Verlag von Hirzel. 1899. S. 221. 
Verhalten der Cervix uteri post partum (Abbildung 2).

Das Präparat stammt von einer Kreissenden, welche wegen Eklampsie bei fünfmarkstückgrossem Muttermund und fast verstrichenem Cervikalcanal durch Accouchement forcé entbunden wurde. Es wurden seichte Incisionen in den äusseren Muttermund gemacht, dann gewendet und ein ausgetragenes Kind entwickelt. Die Wöchnerin starb am dritten 7 age post partum infolge der Eklampsie.

Ich wählte dieses Präparat, um zu zeigen, wie gross die Regenerationsfähigkeit des Cervixepithels post partum ist. Schon früher hatte ich darauf aufmerksam gemacht, dass das Cervixepithel während der Geburt beim Durchtritt des Kindes nur an der Oberfläche einige Läsionen erleidet, dașs diese aber gewöhnlich sehr schnell post partum sich wieder ausgleichen. Dieser Befund erklärt den Schutz, welchen der Cervikalcanal schon bald post partum gegen Spätinfeetionen im Wochenbett gewinnt.

Im ganzen Verlauf des Bildes sehen wir den Epithelbelag intact und zum Theil schon die Falten des Arbor vitae sich bilden.

Besonders möchte ich noch auf die sehr zahlreichen Hämorrhagien im Cervixgewebe aufmerksam machen, welche keineswegs etwa bloss bei forcirten Entbindungen auftreten, sondern auch, wie schon von anderer Seite ebenfalls betont ist, bei ganz spontanem Geburtsverlauf fast regelmässig zu finden sind, während sie in der Uteruswand vollständig fehlen. Um so merkwürdiger erscheint es, dass trotz dieser sichtbaren Läsionen in den tieferen Gewebstheilen der Cervix das Deckepithel so weitgehend erhalten bezw. so schnell regenerirt ist.

Trennungsfläche der Eihäute von der Uteruswand (Abbildung 3).

Es eignen sich zur Beobachtung hierfür hauptsächlich diejenigen Fälle, bei welchen die Frau in der Nachgeburtsperiode, während noch ein grosser Theil der Placenta und der Eihäute an der Uteruswand festsitzt, stirbt.

Besonders vortheilhaft für die Erhaltung des Gewebes ist es weiterhin, wenn nicht zu starke Blutungen bei der Ablösung der Eihäute aus uterinen Gefässen erfolgt sind.

Der Abbildung 3 liegt der Uterus derselben Kreissenden zu Grunde, von der das erste Präparat stammt, also diejenige, bei 
welcher in der Schwangerschaft im 9. Monat plötzlich der Tod durch Herzinsufficienz eintrat. Es war in Agone der Kaiserschnitt gemacht und das Kind extrahirt. Die Frau starb während der Ausführung des Kaiserschnitts, so dass der Uterus mit anhaftender Placenta in toto gleich exstirpirt wurde. Nach Extraction des Kindes hatte sich der Uterus schon stark zusammengezogen, so dass ein Theil der Eihäute und der Placenta von der Uteruswand abgelöst, während ein anderer Theil noch adhärent war.

Ich wählte zur bildlichen Wiedergabe eine Stelle, wo die Eihaut noch zum Theil an der Uteruswand ansitzt, zum Theil von derselben abgelöst ist.

Das Bild giebt im Allgemeinen die Verhältnisse wieder, welche Langhans und Leopold schon früher beschrieben haben, und ich auf Grund anderer Präparate schon andernorts beschreiben konnte. Wir finden die Trennungsschicht hier im Allgemeinen an der. Grenze von der compacten Zone zur spongiösen; aber, und darin pflichten wir Leopold vollständig bei, die Grenze ist nicht eine absolut scharfe, sondern zum Theil haften, wie in dem Bilde bei e deutlich sichtbar ist, einzelne lockere Gewebsspangen der alveolären Zone noch den abgerissenen Häuten an. Der grössere Theil der alveolären Zone (f) mit den frischen Blutergüssen sitzt aber der stark contrahirten Uterusmusculatur an. Wir erkennen im Bilde ausserdem, wie durch ungleichmässige Zusammenziehung des Uterus bei $f$ und $g$ die spongiöse Zone bei f relativ viel höher ist, als bei g. Bei $f$ sind die Drüsenräume in oft sechsfacher Schicht übereinandergeschoben, während wir bei g, wo offenbar die Zusammenziehung der Uterusmusculatur nicht so stark vor sich gegangen ist, die spongiöse Zone der Decidua nur von geringer Breite finden ${ }^{1}$ ).

Die compacte Zone der Decidua (e) wird von dem Chorionepithel (c) überzogen, welches hier, da die Frau sich noch nicht am Ende der Gravidität befindet, einen relativ breiten Saum darstellt; das stark gewulstete Amnion zeigt an der Oberfläche einen wohlerhaltenen Epithelbelag (b).

Die Drüsenräume der spongiösen Zone der Decidua bei $f$ sind zum grössten Theil auch in den mehr nach der Uterushöhle hin gelegenen Partien mit Epithel ausgekleidet. Das Epithel ist im 9. Monat der Schwangerschaft noch oft in fast continuirlicher

1) 1. c. S. 189 . 
Schicht in den Drüsenräumen nachweisbar; am Ende der Gravidität wird durch die Dehnung der Drüsenräume auch das bekleidende Epithel abgeplattet und an manchen Stellen vollständig abgestossen von der Wand, so dass wir es locker mitten in den Drüsenräumen, zum Theil in Lamellen abgestossen finden.

Von den eröffneten Drüsenschalen aus beginnt die Regencration und.Epithelisirung der Uterusoberfiäche, zum Theil vielleicht auch von den an der Placentarstelle reichlich sichtbaren serotinalen Riesenzellen (Pels Leusden').

Serotinale Riesenzellen im Myometrium der Placentarstelle post partum (Abbildung 4).

Diese Riesenzellen finden sich nur an der Placentarstelle und zwar bis zum 5. Tage post partum. Nach dieser Zeit konnte ich sie, was auch Pels-Leusden, dem wir die beste Beschreibung dieser Gebilde verdanken, erwähnt, in den Präparaten nicht mehr finden. Ich gebe eine mikro-photographische Abbildung der Placentarstelle eines puerperalen Uterus wieder, gewonnen von einer Wöchnerin, welche am Ende der Zeit mit Forceps wegen Eklampsie entbunden wurde. Die Frau starb 18 Stunden post partum an dieser Krankheit.

Ueberall an der Placentarstelle finden wir, zum Theil in Haufen angeordnet, diese syncytialen Zellen mit ihren dunkel gefärbten Kernen, welche in der Decidua und in den obersten Muskellagen liegen. Einen kleinen Abschnitt giebt das Mikrophotogramm wieder. Hier sehen wir diese Zellen meist eine zur Oberfläche der Uterusschleimhaut senkrechte oder schräge Richtung einhalten, indem sie die meist parallel zur Oberfläche gerichteten Muskelbündel in ihrer Verlaufsrichtung kreuzen. Die Zellen liegen zum Theil einzeln, zum Theil durchziehen sie in dichten Reihen das Myometrium, und sind bis zu $1 / 2 \mathrm{~cm}$ unter der Oberfläche der Muskellager zu sehen. An manchen Stellen sind diese Zellen so dicht gelagert, dass sie das ganze Gesichtsfeld beherrschen.

Man kann beim Anblick dieser zahlreichen, an der Placentarstelle sich vorfindenden Gebilde sich kaum des Eindruekes er-

1) Pels Leusden, Ueber die serotinalen Riesenzellen und ihre Beziehungen zur Regeneration der epithelialen Elemente des Uterus und der Placentarstelle. Zeitschr. ‥ Geb. u. Gyn. Bd. 36. S. 1. 
wehren, den Marchandi) folgendermaassen wiedergiebt: "Ein Vorgang; welcher bei der Placentarbildung in so ausgedehntem Maasse stattfindet, wie die Einwanderung der epithelialen Zellen, kann wohl nicht bedeutungslos, nicht zwecklos sein."

Es ist möglich, dass die Ansicht, welche Pels-Leusden auf Grund der mehrfach citirten, unter der Aegide Marchand's entstandenen Arbeit entwickelt, zu Recht besteht, dass die Einwanderung der syncytialen Zellen in die Uteruswandung, speciell die Musculatur, die Bedeutung besitzt, dass daselbst Material aufgespeichert wird, von dem aus die Placentarstelle zum Theil wieder mit neuem Epithel versorgt werden kann. Allerdings hat Aschoff gegen diese Annahme gewichtige Bedenken geltend gemacht.

Im oberen Theile des Bildes finden wir Drüsenräume, welche, wie es auch in der photographischen Wiedergabe zu sehen ist, mit sehr niedrigem Epithel ausgekleidet sind. Auch hier an der Placentarstelle ist eine scharfe Scheidung der Schleimhant vom Muskellager gegeben.

Regeneration der Uterusschleimhaut post partum (Abbildung 5, 6 u. 7).

Zur Regeneration der Uterusschleimhaut post partum möchte ich an dieser Stelle 3 Abbildungen wiedergeben, und zwar vom Endometrium und von der Placentarstelle.

Die Abbildung 5 stellt eine Uterusschleimhat am 5. Tage post partum dar, von einer Wöchnerin, welche in der Mitte des 9. Schwangerschaftsmonats spontan niederkam. Es bestand ein Herzfehler mit starkem Eiweissgehalt des Urins. Die Frau starb plötzlich am 5. Tage post partum während eines stenocardischen Anfalles. Ich habe zur Abbildung deswegen die Schleimhaut des Uterus post partum nach einer Frühgeburt gewählt, weil hier die Drïsensepta breiter sind und die Drüsenräume nicht so weit ausgezogen, so dass die Möglichkeit eines klaren Uebersichtsbildes auf engerem Raum gegeben ist. Im Allgemeinen sind ja die Verhältnisse der Regeneration der Cterusschleimhaut post partum nach einer Frühgeburt in der 35. Woche und am Ende der Zeit dieselben.

1) Marchand, Ueber den Bau der Blasenmole. Zeitschr. f. Geb. u. Gyn. 1895. Bd. 32. H. 3. - Derselbe, Ueber die sogenannten decidualen Geschwülste. Monatsschr. f. Geb. u. Gyn. von Martin u. Saenger. 1895. Bd. 1. H. 5 . 
34 Kroenig, Beitrag zum anatomischen Verhalten der Schleimhaut

Wir sehen in den obersten Partien die epithelbedeckten Drüsensepta eröffnet und zum Theil mit Blutkörperchen und nekrotischen Massen ausgefüllt. In den tieferen Partien sind die Drüsenräume im Allgemeinen vollständig leer. Auch hier erkennt man besonders schön, dass zwar die Schleimhautbalken sich tief in die Musculatur hereinziehen und dieselbe auf eine Strecke hin spalten, dass aber die Grenze zwischen Decidua und Muscularis, wie in allen dergleichen Fällen, überall eine sehr scharfe ist.

Ein ähnliches Verhalten finden wir an der Placentarstelle.

Ich wählte den Uterus von einer Wöchnerin, welche im 9. Monat der Schwangerschaft spontan niedergekommen war. Die Frau starb am 2. Tage post partum an Herzfehler.

In dem Bilde (Abbildung VI) ist auch hier wiederum die Grenze zwischen Decidua und Muscularis ausserordentlich scharf ausgeprägt; man sieht, wie zum Theil die Decidua tief in die Gewebszüge der Muscularis einschneidet. Die Oberfläche ist bedeckt mit grossen Blutthromben und Fibrinmassen, die auf dem Bilde wegen Raummangels zum Theil weggelassen sind; nur ein Theil der in Nekrose begriffenen Decidua konnte wiedergegeben werden. Wir sehen, ähnlich wie am Endometrium, die obersten Drüsenräume eröffnet und zum Theil mit Blıt ausgefüllt; überall ist der Epithelsaum erhalten. Die Drüsenräume, welche der Muscularis zunächst liegen, sind leer, während die mehr nach der Uterushöhle liegenden Drüsenräume mit Blutkörperchen vollgestopft sind.

Betreffs der Einzelheiten verweise ich auch hier wiederum auf die Beschreibung von Leopold in diesem Archiv und auf die Beschreibung eines Theiles dieser Fälle in meiner Monographie.

Nach rechtzeitiger Geburt ist das Verhalten der Placentarstelle post partum im Allgemeinen das gleiche; aus dem angegebenen Grunde habe ich auch hier eine nicht ganz ausgetragene Schwangerschaft bevorzugt.

Von der Placentarstelle nach rechtzeitiger Geburt habe ich nur das oben beschriebene Mikrophotogramm wiedergegeben, da ich die Zahl der Abbildungen natürlich sehr beschränken musste.

Ueber das Verhalten der Uterusschleimhaut in den späteren Wochenbettstagen begnüge ich mich damit, die Stelle eines Endometriums am 18. Tage nach Geburt eines ausgetragenen Kindes hier zu reproduciren (Abbildung VII).

Auch runter normalen Verhältnissen verläuft die Regeneration 
der Schleimhant des Uterus bei verschiedenen Individuen nicht in ganz gleicher Weise. Manchmal sehen wir schon relativ früh die Regeneration der Schleimhaut eintreten, oft jedoch vermissen wir noch in der 3 . bis 4 . Woche den gleichmässigen Epithelbelag der Uterusinnenfläche.

Ich habe von meinen Präparaten dasjenige gewählt, welches nach meiner Meinung die gewöhnlichen Verhältnisse darstellt. Die Wöchnerin war am 18. Tage post partum an croupöser Pneumonie gestorben. Die Abbildung entspricht einer Stelle des Endometriums. Wir sehen die ganze Uterusinnenfläche mit einem gleichmässigen Epithelsaum bedeckt, welcher sich an manchen Stellen in schlauchförmigen Drüsen in die Tiefe einsenkt. Dem Epithelbelag liegt z. Th. locker eine nekrotische Masse mit kleinzelliger Infiltration auf; diese wird gewöhnlich erst eine Zeit lang später abgestossen.

Trotz des continuirlichen Epithelüberzuges können wir von einer vollständigen Regeneration der Uterusschleimhaut natürlich noch nicht sprechen.

Wir haben zwischen Deckepithel und Muskulatur an den verschiedenen Theilen der Uterusinnenfläche eine ganz verschiedene Höhe der Schleimhaut. An manchen Stellen liegt der Epithelbelag ziemlich nahe der Uterusmuskulatur auf, an anderen Stellen ist eine grössere Schicht subepithelialen Gewebes eingeschaltet. Der Reichthum an Drüsen der neugebildeten Schleimhaut ist noch verhältnismässig gering; die schlauchförmigen Drüsen sind im Gewebeschnitt meistens nur eine kurze Strecke hin zu verfolgen. Neben den Drüsenräumen finden sich in der innersten Schleimhautschicht stark erweiterte Capillaren mit deutlichem Endothel, zum grossen Theil aber liegen die rothen Blutzellen frei in den Lücken des netzförmig angeordneten Stromas. Diese subepithelialen Extravasate finden sich fast überall in der Schleimhaut und nehmen den grössten Theil derselben ein. Die Bilder gleichen ziemlich weitgehend der Beschreibung, welche Werth ${ }^{1}$ ) von der Schleimhaut eines myomatösen Uterus 17 Tage post abrasionem giebt (Fall 5 in seiner Arbeit). Bei der Vergrösserung, in welcher die Abbildung gegeben ist, sind diese Blutextravasate im Gewebe sehr deutlich zu erkennen.

1) Werth, Untersuchungen über die Regeneration der Schleimhaut nach Ausschabung der Uteruskörperhöhle. Dieses Arehiv. Bd. 49. S. $370 \mathrm{ff}$. 
An der Placentarstelle ist die Regeneration des Deckepithels noch nicht so weit fortgeschritten; aber auch hier ist z. Th. schon an verschiedenen Stellen ein continuirlicher Epithelbelag zu finden. Ich möchte noch besonders hinzufügen, dass diese Regeneration der Uterusschleimhaut und vor allem des Deckepithels manchmal trotz septischer Processe in gleicher Weise vor sich geht. Besonders bei schwererStreptokokkeninfection ist der primäre Infectionsherd oft wenig verändert und wir können manchmal eine sehr schöne Regeneration der Uterusschleimhaut feststellen. Ich besitze u. a. einen Uterus von einer Wöchnerin, welche im 5. Monat der Schwangerschaft am 10. Tage des Wochenbetts an septischer Peritonitis starb. Die Schleimhaut des Uterus ist hier schon weitgehend mit einer einfachen Lage von Deckepithel überzogen.

Betreffs aller weiteren Einzelheiten verweise ich auf die ausführliche Beschreibung im 2. Theile der „Bacteriologie des weiblichen Genitalkanales".

lch danke Herrn Geheimrath Zweifel für die Ueberlassung des Materials und Herrn Geheimrath Marchand dafür, dass er die Freundlichkeit hatte, die Präparate in Augenschein zu nehmen.

\section{Erklärung der Abbildungen auf Tafel I u. II.}

Figur $I_{1}$. Zeiss, Obj. $a_{2}$, Ocul. 2. Frontalschnitt durch den unteren Theil eines Uterus gravidus Mitte des IX. Schwangerschaftsmonats.

a. Müller'scher Ring, etwas weiter nach links gelegen, im Bilde nicht mehr sichtbar.

b. Stelle an der seitlichen Wand des Uterus, wo die Eihaut mit Decidua reflexa, Chorion und Amnion sich von der Uteruswand abhebt und über den Cervicalcanal ausspannt.

c. Decidua reflexa.

d. Amnionepithel.

e. Chorionepithel.

f. Decidua vera mit wohlausgebildeten Deciduazellen.

Figur $I_{2}$. Die Stelle bei $x$ in Fig. $I_{1}$ bei stärkerer Vergrösserung (Zeiss, Object $\mathrm{E}$, Ocul. 2) wiedergegeben, um das Cervixepithel bei $g$ und das niedrige lrubische Epithel bei $h$, welches der Eihaut an der freien Fläche aufsitzt, zu zeigen.

Figur $\mathrm{I}_{3}$. Uebersichtsbild in natürlicher Grösse. 
der Cervix und des Uterus während der Schwangerschaft etc.

a. Grenze zwischen unterem und oberem Abschnitt der Cerrix (Müller'scher Ring).

a.-b. Trichterförmig erweiterter Cervixcanal.

b. Abgangsstelle der Eihaut.

c. Decidua vera.

d. Ueber den Cervicalcanal ausgespannte Eihaut.

Figur II. Zeiss, Obj. $\mathrm{a}_{2}$, Ooul. 4. Schnitt aus der Cervix am 3. Tage post partum.

a. Cervixepithel.

b. Hämorrhagien im Cervixgewebe.

Figur III. Zeiss, Obj. a $a_{2}$, Ocul. 2. Trennungsfläche der Eihaut von der Uteruswand. Sectio caesarea. Mitte des IX. Schwangerschaftsmonats.

a. Beginn der Ablösung der Eihaut an der Grenze von compacter Zone zur spongiösen Zone der Decidua.

b. Amnion.

c. Chorion.

e. Compacte Zone der Decidua.

f. u. g. Alveoläre Zone der Decidua.

Figur IV. Mikrophotogramm. Placentarstelle 18 Std. post partum.

Figur V. Zeiss, Obj. A, Ocul. 2. Endometrium am 5. Tage post partum. Frühgeburt Mitte des IX. Schwangerschaftsmonats.

Figur VI. Zeiss, Obj. A, Ocul. 2. Placentarstelle am 2. Tage post partum. Geburt Mitte des IX. Schwangerschaftsmonats.

a. Muscularis.

b. Decidua z. Thl. nekrotisch.

Figur VII. Zeiss, Obj. A, Ocul. 2. Endometrium nach ausgetragener Schwangerschaft am 18. Tage post partum. 
¿
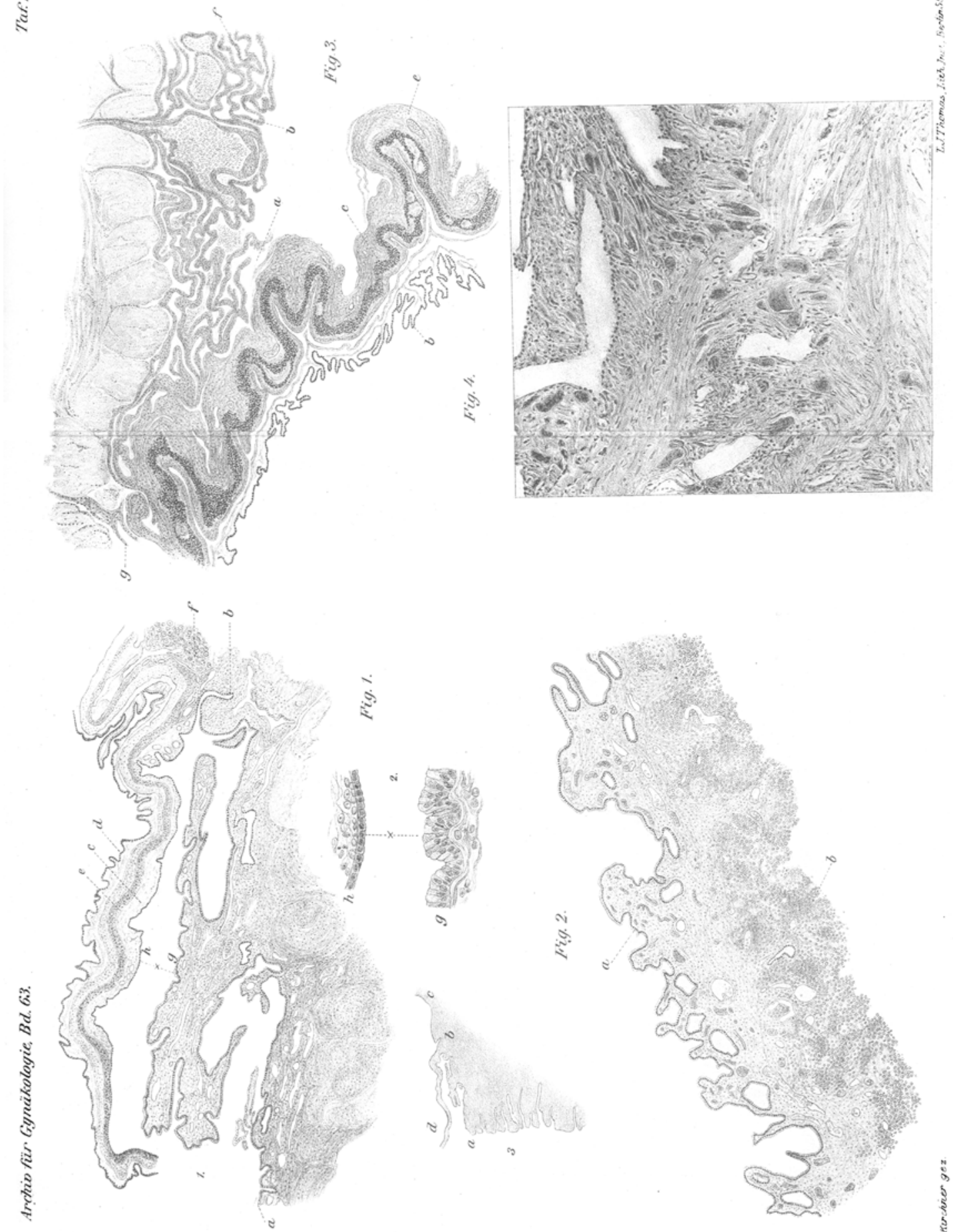

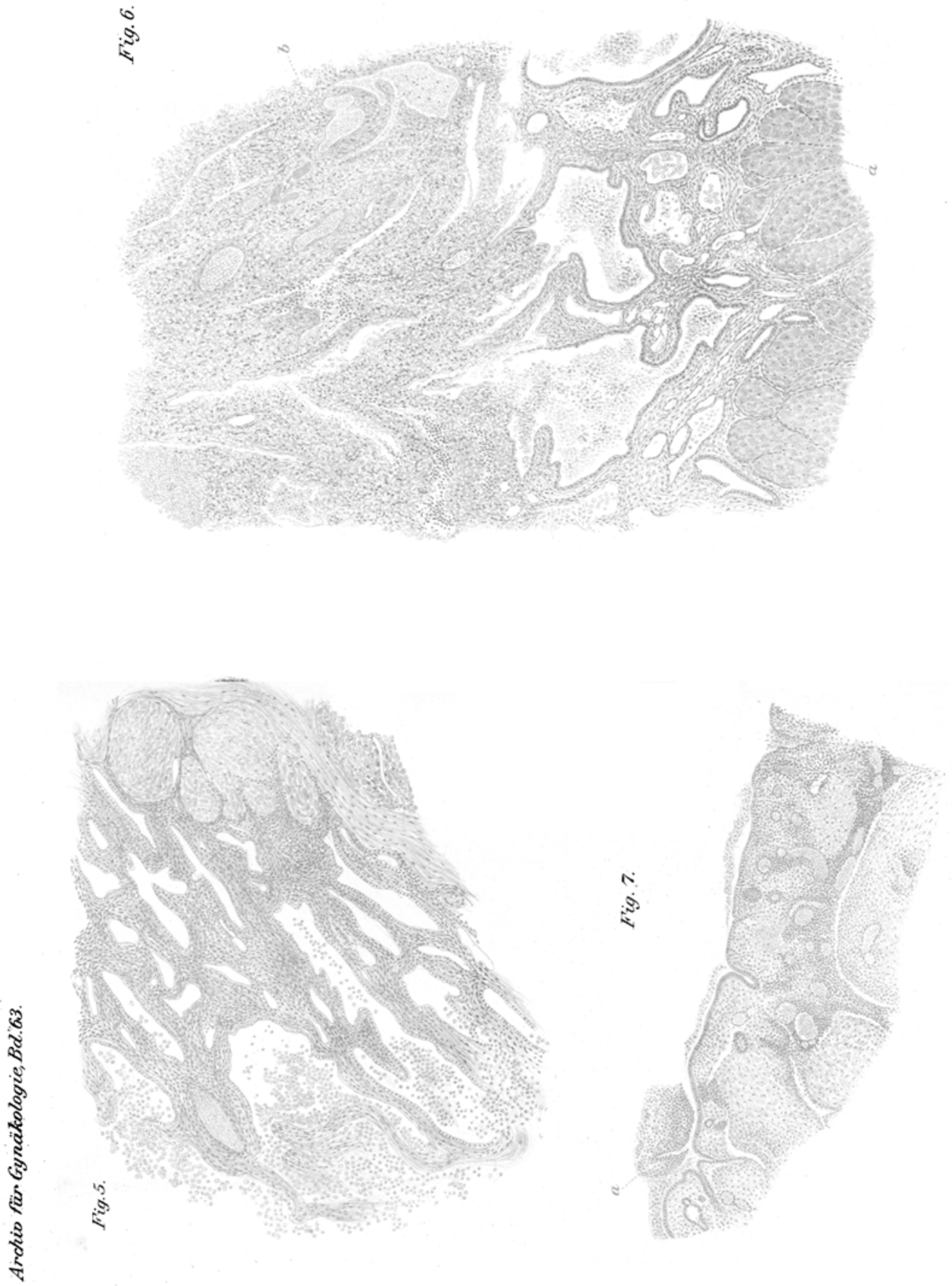